\title{
Measurements of NaI(Tl) Electron Response: Comparison of Different Samples
}

\author{
Giulia Hull, Woon-Seng Choong, Member, IEEE, William W. Moses, Fellow, IEEE, Gregory Bizarri, John D. \\ Valentine, Senior Member, IEEE, Stephen A. Payne, Nerine J. Cherepy and Bryan W. Reutter
}

\begin{abstract}
This paper measures the sample to sample variation in the light yield proportionality of $\mathrm{NaI}(\mathrm{Tl})$, and so explores whether this is an invariant characteristic of the material or whether it depends on the chemical and physical properties of the tested samples.

We report on the electron response of nine crystals of $\mathrm{NaI}(\mathrm{Tl})$, differing in shape, volume, age, manufacturer and quality. The proportionality has been measured at the SLYNCI facility in the energy range between 3.5 to $460 \mathrm{keV}$. We observe that while samples produced by the same manufacturer at approximately the same time have virtually identical electron response curves, there are significant sample to sample variations among crystals produced by different manufacturers or at different times.

In an effort to correlate changes in the electron response with details of the scintillation mechanism, we characterized other scintillation properties, including the gamma response and the $x$ ray excited emission spectra and decay times, for the nine crystals. While sample to sample differences in these crystals were observed, we have been unable to identify the underlying fundamental mechanisms that are responsible for these differences.
\end{abstract}

\section{INTRODUCTION}

$\mathrm{I}_{\mathrm{s} e}^{\mathrm{T}}$ has been observed that the luminous efficiency for most scintillators depends on the excitation energy. At present, there is a general consensus that this effect, which is known as non-proportionality, is a key contributor to the degradation of energy resolution [1]-[4].

A powerful way to study the electron response of a scintillator is the Compton Coincidence Technique (CCT) introduced by Valentine and Rooney, [5], [6]. The crystal under study is irradiated with a well-collimated ${ }^{137} \mathrm{Cs}$ source and when a Compton scatter occurs in the scintillator, the scattered electron, which is absorbed within the crystal, is detected in coincidence with the secondary gamma ray. In this way it is possible to relate the amount of light produced in the crystal to the energy of the Compton scattered electron that can be evaluated knowing the energy of the secondary gamma. A wide range of electron energies in the scintillator can be

Manuscript received December 3, 2007. This work was supported by the Domestic Nuclear Detection Office in the Department of Homeland Security and by the National Nuclear Security Administration, Office of Defense Nuclear Nonproliferation, Office of Nonproliferation Research and Development (NA-22) of the U.S. Department of Energy under Contract No. DE-AC02-05CH11231, and this work was performed under the auspices of the U.S. Department of Energy by Lawrence Livermore National Laboratory under contract No. DE-AC52-07NA27344.

G. Hull, J. D. Valentine, S. A. Payne, N. J. Cherepy are with Lawrence Livermore National Laboratory, Livermore, CA 94551 USA (telephone: +1925-423-0570,e-mail: payne3@1lnl.gov).

W. -S. Choong, W. W. Moses, G. Bizarri and B. W. Reutter are with Lawrence Berkeley National Laboratory, Berkeley, CA 94720 USA. investigated by varying the angle of detection $\theta$ of the secondary gamma rays [5], [6].

The original design of the Compton Coincidence instrument was limited by a very low counting rate, which necessitated several weeks of data collection time for the characterization of a single sample. For this reason, only a few scintillator materials have had their electron response measured with the CCT method [6]-[10], and in particular, only one crystal of each material was studied.

As significant sample to sample variations in other scintillation properties (e.g., light output and decay time [11]) have been observed in some materials, we feel that it is important to determine whether the proportionality is an inherent, sample-independent property of the scintillator or it depends on the specimen tested.

Therefore, we measure the electron response of several different samples of $\mathrm{NaI}(\mathrm{Tl})$. We choose to study $\mathrm{NaI}(\mathrm{Tl})$ not only because this material is the most widely used inorganic crystal and is considered as a reference detector material for gamma spectroscopy, but also because it has been known for over sixty years and has been in commercial production for much of that time, and so its production should be relatively stable.

\section{EQUIPMENT AND METHODS}

\section{A. The SLYNCI Facility}

The work was performed with the Scintillation Light Yield Non-proportionality Characterization Instrument (SLYNCI), a second-generation Compton Coincidence device. This newly developed instrument acquires data roughly two orders of magnitude faster than the original setup, allowing many scintillator samples to be tested. The apparatus, which is described more completely in [12], employs five high purity germanium detectors, a collimated $1 \mathrm{mCi}$ Cs-137 source and a highly linear XP2060B Photonis photomultiplier tube. Each HPGe subtends an angle of about $30^{\circ}$ and thus detects a broad range of Compton scattered gamma energies. The radioactive source is placed on a rotating support so that the angle of the incident $662 \mathrm{keV}$ gamma ray irradiating the crystal can be rotated by $15^{\circ}$. In this way, with two source positions, it is possible to investigate the electron response of the scintillator in the full energy range between 3.5 to $460 \mathrm{keV}$.

A validation study is reported in [13], and includes the electron response of two samples of $\mathrm{NaI}(\mathrm{Tl})$. 


\section{B. The tested $\mathrm{NaI}(\mathrm{Tl})$ crystals}

In this work we tested nine $\mathrm{NaI}(\mathrm{Tl})$ samples, which present differences in shape, volume, age, manufacturer and quality; in table 1, a detailed list of the tested crystals is reported. They include four sets of "paired" crystals, i.e. crystals that were procured at the same time from the same vendor, and so should have nearly identical scintillation properties, (\#568 and \#569, \#570 and \#571, \#572 and \#573, \#578 and \#579) plus one unpaired crystal (\#574).

TABLE I

THE TESTED CRYSTALS

\begin{tabular}{|ccccccc|}
\hline $\begin{array}{c}\text { Sample } \\
\text { ID }\end{array}$ & Shape & Dimensions & Manufacture & Age & Quality \\
$\# 568$ & Cylinder & $0.5 " \varnothing \times 0.5 "$ & Saint-Gobain & New & $\mathrm{R}<10 \% @ 122 \mathrm{keV}$ \\
$\# 569$ & Cylinder & $0.5 " \varnothing \times 0.5 "$ & Saint-Gobain & New & $\mathrm{R}<10 \% @ 122 \mathrm{keV}$ \\
$\# 570$ & Cylinder & $0.5 " \varnothing \times 0.5 "$ & Saint-Gobain & $\mathrm{New}$ & Standard \\
$\# 571$ & Cylinder & $0.5 " \varnothing \times 0.5 "$ & Saint-Gobain & New & Standard \\
$\# 572$ & Cube & $1 \mathrm{~cm}^{3}$ & Scionix & Old & Yellowed \\
$\# 573$ & Cube & $1 \mathrm{~cm}^{3}$ & Scionix & Old & Yellowed \\
$\# 574$ & Cylinder & $1 " \varnothing \times 1 "$ & Harshaw & Old & Standard \\
$\# 578$ & Cylinder & $1 \mathrm{~cm} \varnothing \times 1 \mathrm{~cm}$ & ScintiTech & New & Standard \\
$\# 579$ & Cylinder & $1 \mathrm{~cm} \varnothing \times 3 \mathrm{~cm}$ & ScintiTech & New & Standard \\
\hline
\end{tabular}

In particular, we characterized the response of four $\mathrm{NaI}(\mathrm{Tl})$ crystals from Saint-Gobain, of identical shape and volume that were purchased at the same time; for two of them we requested a particularly good $(<10 \%$ fwhm $)$ energy resolution at $122 \mathrm{keV}$, while the other two are standard Saint-Gobain production. We also tested two old Scionix crystals, which were purchased in 1997 and had yellowed, an old, large Harshaw sample, and finally two new ScintiTech crystals with the same diameter but different volumes.

\section{THE ELECTRON RESPONSE}

To evaluate the electron response for the nine $\mathrm{NaI}(\mathrm{Tl})$ samples under study, we acquired coincidence events between the PMT and the HPGe detectors. For each collimator position we acquired 6 million events, including both single and coincidence events, for a collection data time between 10 to 12 hours, depending on the volume of the samples. The acquired single events were used for on-line calibration of the PMT and the HPGe detector positioned directly in front of the collimator. This detector samples the gamma rays scattered at small angles and thus measures the low electron energies, for this reason it is particularly sensitive to gain drift variation.

In fig. 1 the relative light yield for the nine $\mathrm{NaI}(\mathrm{Tl})$ crystals under study is reported as a function of the electron energy. The data distributions are normalized at the energy value of $450 \mathrm{keV}$. This plot clearly highlights that there are sample-tosample variations in the electron response of $\mathrm{NaI}(\mathrm{Tl})$ for different crystals. In particular, it is interesting to observe that we measured a $10 \%$ difference in the low energy range for sample \#568 (round full symbol) and \#570 (square full symbol), which have the same shape and volume, and are produced from the same manufacturer. However the light yield data distributions for "paired" crystals, i.e. crystals having the same manufacturer and specifications, consistently match through the whole energy range, for example $\mathrm{NaI}(\mathrm{Tl})$ \#568 (round full symbol) and \#569 (round empty symbol). Since the acquisition run for $\mathrm{NaI}(\mathrm{Tl}) \# 569$ was performed two weeks after that of sample \#568, the agreement of these data distributions indicates that the apparatus is stable with time.

Fig. 1 also shows the $\mathrm{NaI}(\mathrm{Tl})$ electron response measured by Rooney and Valentine [6]. While its relative light yield is consistently above those from the other samples at low energy, the error bars from this measurement are larger than those from the SLYNCI apparatus, we believe that this difference is also attributable to sample to sample variations. 


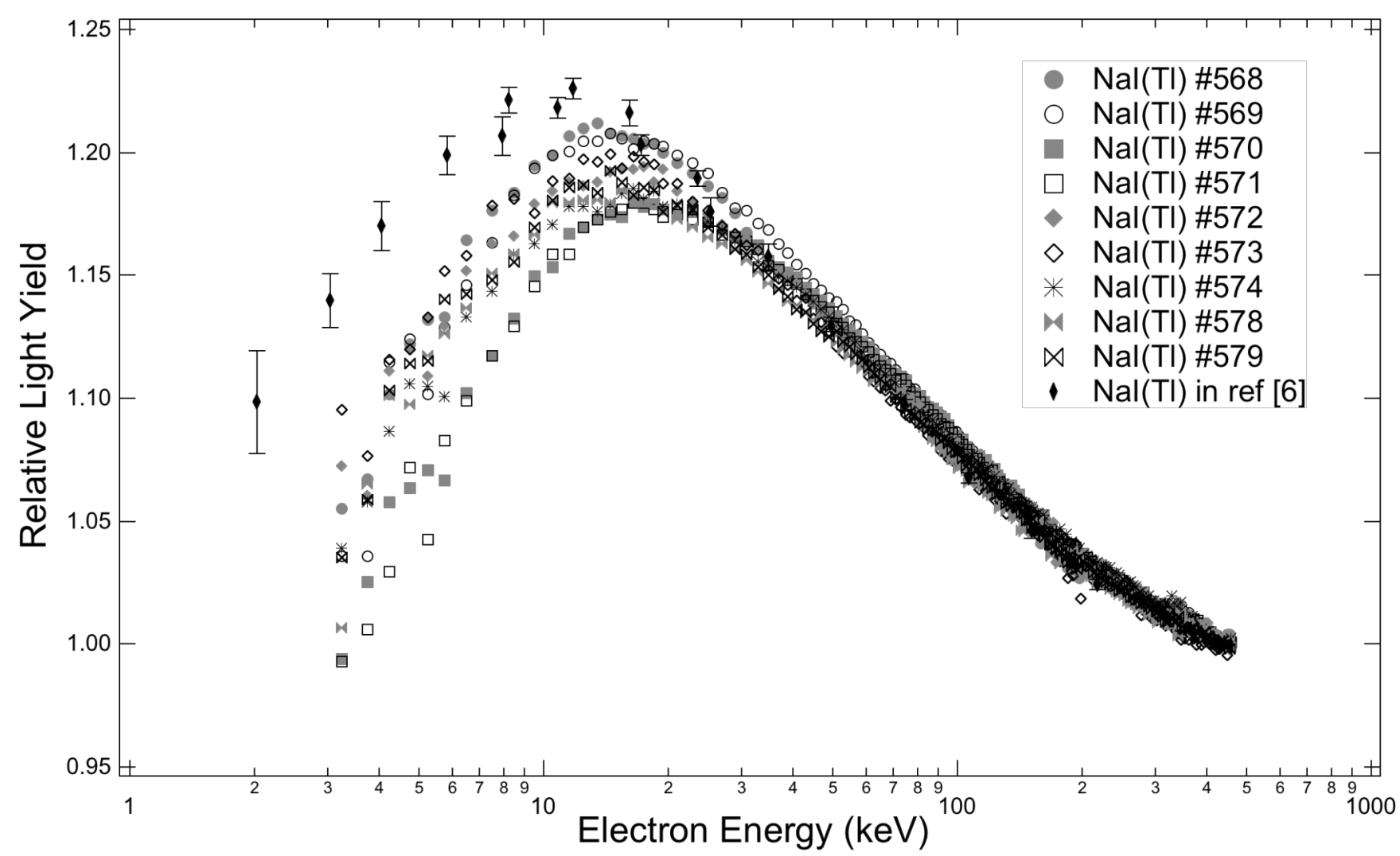

Fig. 1. Electron response, normalized at $450 \mathrm{keV}$, for the nine $\mathrm{NaI}(\mathrm{Tl})$ under study. The NaI(Tl) relative light yield measured by Rooney and Valentine [6] is reported also for comparison. For the entire energy range, the electron response is measured with accuracy better than $1 \%$, [13].

\section{The GAMMA-RAY ReSPONSE}

The gamma ray response of the nine $\mathrm{NaI}(\mathrm{Tl})$ crystals was tested using five radioactive sources $\left({ }^{109} \mathrm{Cd},{ }^{133} \mathrm{Ba},{ }^{241} \mathrm{Am},{ }^{57} \mathrm{Co}\right.$ and ${ }^{137} \mathrm{Cs}$ ), emitting gamma rays in the energy range between $22.5 \mathrm{keV}$ to $661.657 \mathrm{keV}$. We acquired these pulse height spectra with the same experimental set-up employed for the electron response measurements, operated in singles mode; for this study, we furthermore blocked the $1 \mathrm{mCi}^{137} \mathrm{Cs}$ source, so that the strong flux of $662 \mathrm{keV}$ gamma rays did not interfere with the pulse height measurements.

In order to evaluate the light yield and the FWHM (full width at half maximum) energy resolution as a function of the gamma ray energy, we performed a Gaussian fit procedure on the main emission peaks of the acquired pulse height spectra to estimate the centroid and the width. For the photopeaks from X-rays emitted by ${ }^{109} \mathrm{Cd}$ and ${ }^{133} \mathrm{Ba}$, which can not be individually resolved, we applied a weighted average method to determine the centroid and the width of the associated peaks.

Even allowing for the differences in volume, packaging and coupling to the PMT, for the nine tested $\mathrm{NaI}(\mathrm{Tl})$ samples we observed quite large differences in the brightness, up to a factor of $\sim 1.6$. In fig. 2, the measured light yield, normalized at $662 \mathrm{keV}$, is presented as a function of the gamma radiation energy. As expected we can observe an increase of the light yield with decreasing energy, and the drop around $30 \mathrm{keV}$ corresponding to the Ka edge of iodine, [14],[15].
As we already observed for the electron response, "paired" crystals, i.e. \#568 and \#569, \#570 and \#571, \#572 and \#573, \#578 and \#579 showed comparable light yield data distributions as a function of the energy.

From the acquired pulse height spectra we also evaluated the FWHM energy resolution of the crystals.

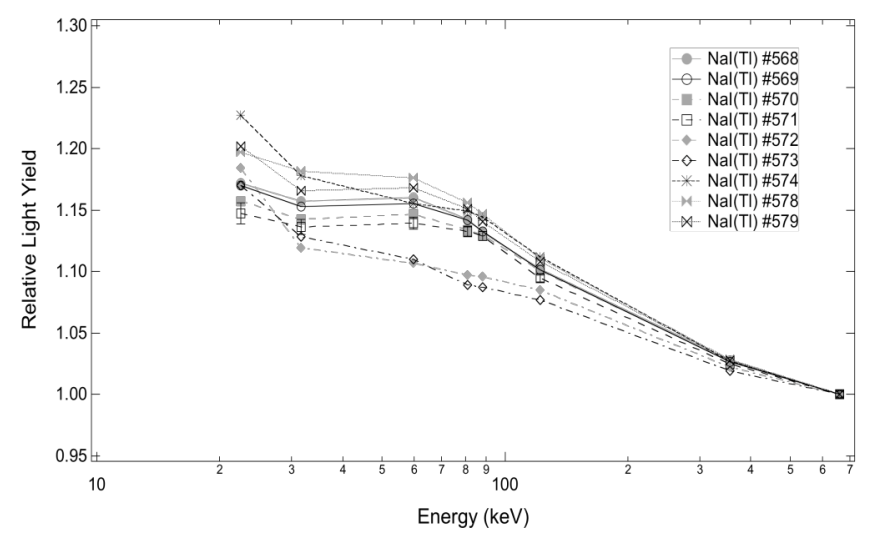

Fig. 2. Relative light yield as a function of the gamma energy for the nine $\mathrm{NaI}(\mathrm{Tl})$ samples under study. The error bars on the measured light yield, here reported for the crystal \#571 as an example, are of the same order for all the tested crystals. 


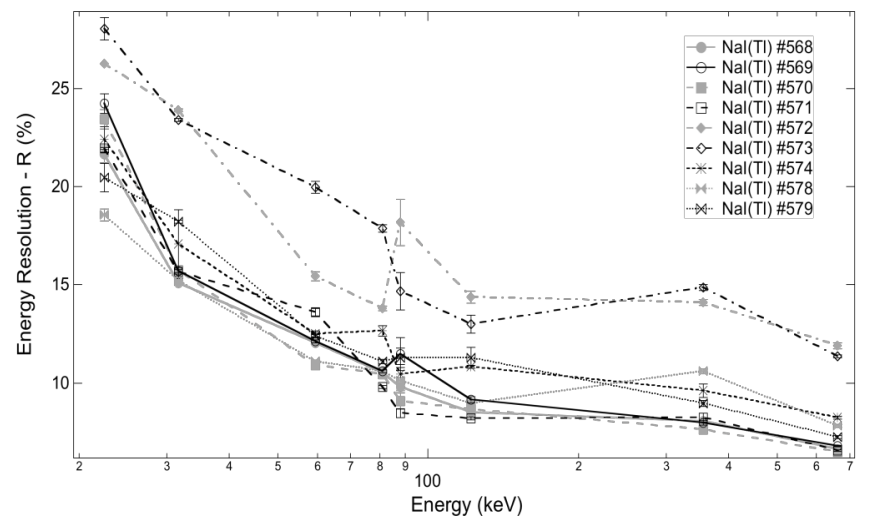

Fig. 3. FWHM energy resolution as a function of the gamma energy for the nine $\mathrm{NaI}(\mathrm{Tl})$ samples under study.

At $662 \mathrm{keV}$, the Saint-Gobain crystals (\#568, \#569, \#570, \#571) provided energy resolution of $6.5-6.8 \%$, while for the yellowed Scionix samples (\#572, \#573) we measured a value slightly worse than $11 \%$, clearly demonstrating the degraded quality of these crystals. In fig. 3 the measured energy resolution is reported as a function of the gamma-ray energy. For all the investigated crystals the error bars for the data point at $88 \mathrm{keV}$ were larger than the neighboring measurements; This effect is essentially due to weak ${ }^{109} \mathrm{Cd}$ line at $88 \mathrm{keV}$ that results in a lower collection of statistics in the full absorption peak at this energy value. All the investigated crystals showed a flattening of the data distribution in the energy range between $100 \mathrm{keV}$ and $400 \mathrm{keV}$, in agreement with the step like distribution typical of $\mathrm{NaI}(\mathrm{Tl})$ observed in other works, [3], [16]. This effect is mainly due to the intrinsic contribution to the energy resolution that becomes significant for energies higher than $100 \mathrm{keV}$ and dominates above $300 \mathrm{keV}$.

\section{X-RAY EXCITED EMISSION SPECTRA}

Emission spectra of the nine $\mathrm{NaI}(\mathrm{Tl})$ under study were measured at room temperature, irradiating the samples with an $\mathrm{x}$-ray beam characterized by a mean energy of $30 \mathrm{keV}$ (peak energy at $50 \mathrm{keV}$ ) and an intensity of $20 \mathrm{~Gy} / \mathrm{min}$. The $\mathrm{x}$-ray excited scintillation was collected into a SpectraPro-2150i spectrometer (Acton Research Corp.) coupled to a PIXIS:100B charge-coupled detector (Princeton Instruments Inc.). The $\mathrm{CCD}$ is thermoelectrically cooled to $-70^{\circ} \mathrm{C}$ and has a $1340 \times 100$ pixel array. For each crystal sample the background was first measured with the CCD shutter closed; then we acquired a blue spectrum (between 200 and $360 \mathrm{~nm}$ ), a green spectrum (between 360 and $620 \mathrm{~nm}$ ), and finally a spectrum in the red region (between 620 and $1000 \mathrm{~nm}$ ). More details on the experimental set-up and procedure are reported in [17].

We observed very similar emission spectra for samples produced by the same manufacturer. In fig. 4 the spectra acquired for three of the tested $\mathrm{NaI}(\mathrm{Tl})$ crystals are presented: dotted line for sample \#568 by Saint-Gobain, dashed line for sample \#572 by Scionix (yellowed crystal), and full line for sample $\# 578$ by ScintiTech. All the spectra have been corrected for the wavelength-dependent detection efficiency of the set up. Fig. 4 also shows the undoped $\mathrm{NaI}$ emission spectrum measured by Moszynski et al. [18], showing a pure exciton band; The comparison with this spectrum clearly indicates that the satellite peaks observed in the spectra acquired in this work are not due to the exciton band typical of undoped $\mathrm{NaI}$ that appears at $310 \mathrm{~nm}$.

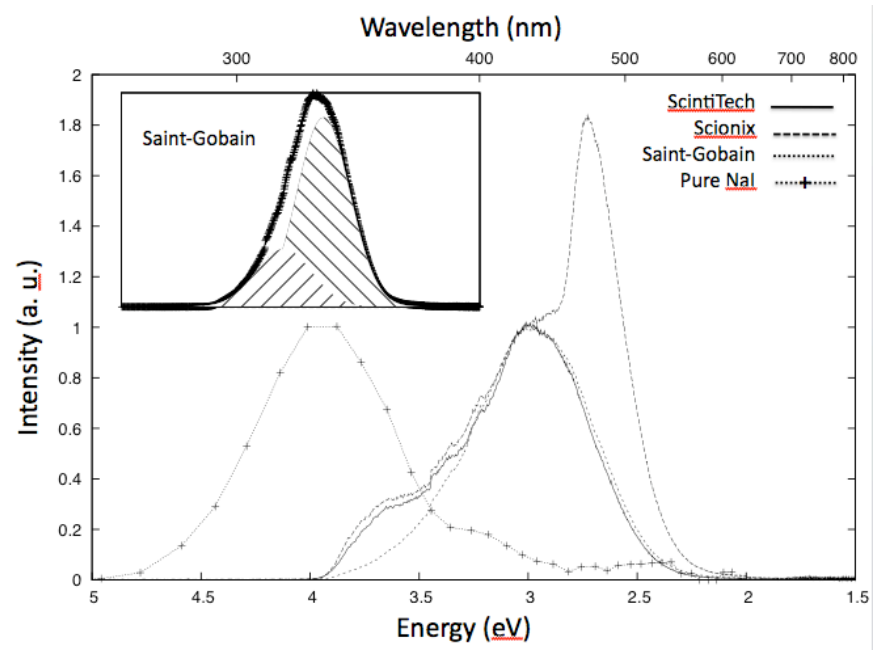

Fig. 4. X-ray excited emission spectra for three $\mathrm{NaI}(\mathrm{Tl})$ samples. The emission spectrum for pure NaI as measured by Moszynski et al. (ref. [18]) is reported for comparison. In the insert the Gaussian fit for the Saint-Gobain crystal \#568 is presented, as an example.

To evaluate the different components in each spectrum, we performed a multiple Gaussian fit on the data distributions. For the Saint-Gobain crystals we observed two bands: a main peak centered at $2.92 \mathrm{eV}(420 \mathrm{~nm})$ that we attribute to the thallium emission and a weaker satellite peak centered at 3.28 $\mathrm{eV}(380 \mathrm{~nm})$ that is presumed to be due to impurities. The satellite component shows an intensity of $20 \%$ compared to the main $\mathrm{Tl}$ band. The ScintiTech samples show the same two peaks at 2.92 and $3.28 \mathrm{eV}$, plus an additional component at $3.64 \mathrm{eV}(340 \mathrm{~nm})$; the two satellite peaks have intensities equal to $40 \%$ and $15 \%$ compared to the main band, respectively. For the Scionix yellowed samples the multipleGaussian fit estimated the presence of the peaks at $2.92 \mathrm{eV}$, $3.28 \mathrm{eV}$ and $3.64 \mathrm{eV}$ and an additional strong emission at 2.70 $\mathrm{eV}(480 \mathrm{~nm})$.

\section{DeCay Time Measurements}

The decay time measurements were performed by irradiating the $\mathrm{NaI}(\mathrm{Tl})$ crystals with a pulsed x-ray beam of mean energy of $19 \mathrm{keV}$. The peak energy is $30 \mathrm{kV}$ and a 0.51 $\mathrm{mm}$ thick Aluminum foil is used to reduce the presence of soft $\mathrm{x}$-rays in the beam. Details on the experimental set-up are reported in [19].

Fig. 5 shows the decay signal acquired for $\mathrm{NaI}(\mathrm{Tl})$ sample $\# 568$, as an example. In order to estimate the decay time from the acquired signals we performed a fit procedure on the data distribution with the function: 


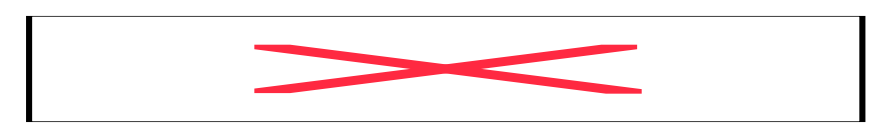

This fitting function takes into account two different mechanisms for the excitation of the thallium ions according to the same approach described by Kubota and coauthors in [20] to fit the flat top in the decay curves of $\mathrm{NaI}(\mathrm{Tl})$. The first component of the fitting function represents the prompt excitation via sequential capture of electron-hole pair, the second component represents the delayed excitation via the energy transfer from Self Trapped Excitons (STE) to the thallium site.

When we fit these curves to a single exponential decay, we obtain a decay value of approximately $230 \mathrm{~ns}$, consistent with the more commonly reported values. However, the chisquared for the single exponential fit is considerably worse.

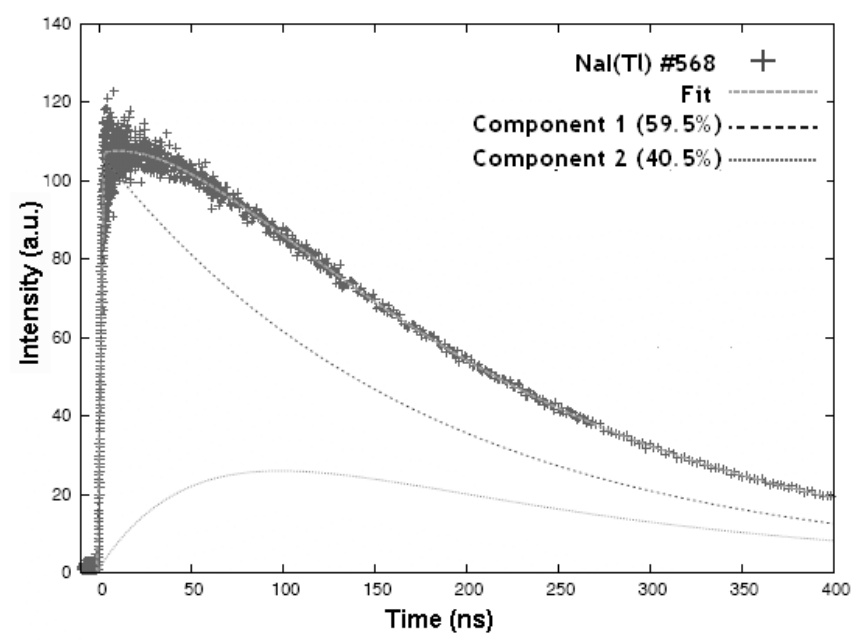

Fig. 5. Decay time signal for $\mathrm{NaI}(\mathrm{Tl}) \# 568$.

For all of the tested $\mathrm{NaI}(\mathrm{Tl})$ crystals we measured nearly identical values for the prompt decay time $T$ (typically 173 ns) and the rise time $T_{r}$ (59 ns). However, we observed large differences in the ratio between $\mathrm{A}_{1}$ and $\mathrm{A}_{2}$. As an example, for $\mathrm{NaI}(\mathrm{Tl}) \# 568$ we measured a ratio of $59.5 \%$ for the prompt component, while it becomes more significant for $\mathrm{NaI}(\mathrm{Tl}) \# 578$, being the $79 \%$ of the total decay signal.

\section{DISCUSSION}

The aim of this paper was to study the sample-to-sample variation in the electron response of $\mathrm{NaI}(\mathrm{Tl})$ and so to assess whether the proportionality is an intrinsic property of the material or it depends on the particular crystal under study. We clearly observed variation in both the electron response and the gamma response in nine different $\mathrm{NaI}(\mathrm{Tl})$ samples. Given the similar behavior of "paired" crystals (i.e., crystals having same shape and volume and produced from the same manufacturer), we believe that the observed variations are not scatter in the experimental measurements but are due to intrinsic characteristics of the tested samples.
The x-ray excited emission spectra and decay time measurements of the nine $\mathrm{NaI}(\mathrm{Tl})$ specimens provided similar results: "paired" crystals exhibit very similar behavior while significant differences in the response are measured for "unpaired" samples. The presence of multiple emission peaks and decay times indicates that there are a minimum of two types of luminescent center in $\mathrm{NaI}(\mathrm{Tl})$, and arguably up to four types. However, the correspondence between emission spectra, decay time, and luminescent center type is unclear. For example, the samples have two, three, or four emission peaks (indicating up to four different luminescent center types), yet a maximum of two different time constants are measured in each sample (suggesting that there are only two luminescent center types).

Similarly, we do not observe any correlation between the electron response measurements shown in fig. 1 and these other data. There is variation between the samples, mostly for electron energies below $20 \mathrm{keV}$, but we do not observe correlation between the low energy electron response and the decay time or emission spectra.

\section{CONCLUSIONS}

The shape of the electron response, measured for nine $\mathrm{NaI}(\mathrm{Tl})$ samples, is qualitatively similar for all samples but showed up to $10 \%$ sample-to-sample variation. We also observed large differences in the gamma ray light yields, x-ray excited emission spectra and ratio of the decay components on the tested crystals. As the measurements on "paired" crystals (which are likely to have been grown under the same conditions and could even originate from the same boule) were nearly identical, we believe that these differences cannot be attributed to measurement error.

Thus, we conclude that the electron response is not a fundamental property of $\mathrm{NaI}(\mathrm{Tl})$, but has sample-to-sample variations. This is not completely surprising in light of the sample-to-sample variations that we observe in the emission spectrum and decay time. What perhaps is more surprising is the relative lack of uniformity in $\mathrm{NaI}(\mathrm{Tl})$ samples, given the many years that this material has been produced commercially. While it is certainly possible that other scintillator materials may exhibit very small sample-to-sample variations in their electron response, these results also suggest that it would be prudent to measure the proportionality for multiple samples of the same material rather than assume that measurements made on a single sample represent an intrinsic property of the material.

The origins of non-proportionality are not known, and so the origin of sample-to-sample variations in nonproportionality is an even larger mystery. However, our thoughts are that non-proportionality is caused by competition for excitation, especially competition between the luminescent center(s) and non-luminescent traps. The concentrations and types of impurities are likely to vary sample by sample, and if the luminescence or trapping depends on these impurities, the "balance" that results from this competition can change. This can affect the proportionality, and can also affect the other scintillation properties such as the emission spectra and decay 
time. Thus, for materials that are sensitive to impurities, which we assume includes $\mathrm{NaI}(\mathrm{Tl})$, one would expect sample to sample differences in most of the scintillation properties. However, it is also conceivable that some materials are insensitive to impurities, and so would show little sample to sample variation.

\section{ACKNOWLEDGMENTS}

We would like to thank Dr. Stephen E. Derenzo of LBNL for the X-ray measurements. This work is supported in part by the National Nuclear Security Administration, Office of Defense Nuclear Nonproliferation, Office of Nuclear Nonproliferation Research and Engineering (NA-22) of the U.S. Department of Energy under contract No. DE-AC0205CH11231, grant number NNSA LB06-316-PD05 / NN2001000, and in part under the auspices of the U.S. Department of Energy Lawrence Livermore National Laboratory under Contract DE-AC52-07NA27344 and by the Domestic Nuclear Detection Office of the Department of Homeland Security.

\section{REFERENCES}

[1] C.D. Zerby, A. Meyer and R.B. Murray, "Intrinsic line broadening in $\mathrm{NaI}(\mathrm{Tl})$ gamma-ray spectrometers", Nucl. Instr. \& Meth. vol. 12, pp. 115-123, Mar. 1961.

[2] P. Dorenbos, J.T.M. De Haas and C.W.E. van Eijke, "Nonproportionality in the scintillation response and the energy resolution obtainable with scintillation crystals ", IEEE Trans. Nucl. Sci., vol. 42 , no. 6, pp. 2190-2202, Dec. 1995 .

[3] M. Moszynski, J. Zalipska, M. Balcerzyk, M. Kapusta, W. Mengesha, J.D. Valentine, "Intrinsic energy resolution of $\mathrm{NaI}(\mathrm{Tl}) "$, Nucl. Instr. \& Meth. A vol. 484, pp. 259-269, 2002.

[4] M. Moszynski, "Inorganic scintillation detectors in $\gamma$-ray spectrometry", Nucl. Instr. \& Meth. A vol. 505, pp. 101-110, 2003.

[5] J.D. Valentine and B.D. Rooney, "Design of a Compton spectrometer experiment for studying non-linearity and intrinsic energy resolution", Nucl. Instr. \& Meth. A vol. 535, pp. 37-40, Dec. 1994.

[6] B.D. Rooney and J.D. Valentine, "Benchmarking the Compton Coincidence Technique for measuring electron response nonproportionality in inorganic scintillator ", IEEE Trans. Nucl. Sci., vol. 43, no. 3, pp. 1271-1276, Jun. 1996.

[7] T.D. Taulbee, B.D. Rooney, W. Mengesha, J.D. Valentine, "The measured electron response nonproportionalities of $\mathrm{CaF}_{2}, \mathrm{BGO}$, and LSO", IEEE Trans. Nucl. Sci., vol. 44, no. 3, pp. 489-493, Jun. 1997

[8] W. Mengesha, T.D. Taulbee, B.D. Rooney, J.D. Valentine, "Light yield nonproportionality of CsI(Tl), CsI(Na), and YAP", IEEE Trans. Nucl. Sci., vol. 45, no. 3, pp. 456-461, Jun. 1998.

[9] W. Mengesha, T.D. Taulbee, J.D. Valentine, B.D. Rooney, " $\mathrm{Gd}_{2} \mathrm{SiO}_{5}\left(\mathrm{Ce}^{3+}\right)$ and $\mathrm{BaF} 2$ measured electron and photon responses", Nucl. Instr \& Meth A, vol. 486 (1-2), pp.448-452, Jun. 2002.

[10] E.V.D. van Loef, W. Mengesha W, J.D. Valentine, P. Dorenbos, C.W.E. van Eijk, "Non-proportionality and energy resolution of a $\mathrm{LaCl}_{3}: 10 \%$ $\mathrm{Ce}^{3+}$ scintillation crystal", IEEE Trans. Nucl. Sci., vol. 50, Issue 1, pp. 155-158, Feb. 2003.

[11] C.L. Melcher, M. Schmand, M. Eriksson, L. Eriksson, M. Casey, R. Nutt, J.L. Lefaucheur, and B. Chai, "Scintillation Properties of LSO:Ce Boules", IEEE Trans. Nucl. Sci., vol. 47, No. 3, pp. 965-968, Jun. 2000.

[12] W.-S. Choong, K. M. Vetter, W.W. Moses, G. Hull, S.A. Payne, N.J. Cherepy, and J.D. Valentine, "Design of a facility for measuring scintillator non-proportionality", IEEE Trans. Nucl. Sci., vol. 55, no. 3, pp. 1753-1758, Jun. 2008.

[13] W.-S. Choong, G. Hull, W.W. Moses, K. M. Vetter, S.A. Payne, N.J. Cherepy, and J.D. Valentine, "Performance of a facility for measuring scintillator non-proportionality", IEEE Trans. Nucl. Sci., vol. 55, no. 3, pp. 1073-1078, Jun. 2008.
[14] B.D. Rooney and J.D. Valentine, "Scintillator light yield nonproportionality: Calculating photon response using measured electron response", IEEE Trans. Nucl. Sci., vol. 44, no. 3, pp. 509-516, Jun. 1997.

[15] G. F. Knoll, "Radiation detection and measurement", Third Edition, John Wiley and Sons, Inc. 2000.

[16] C.W.E. van Eijk, P. Dorenbos, E.V.D. van Loef, K. Kiamer, H.U. Gudel, "Energy resolution of some new inorganic-scintillator gammaray detectors", Rad. Meas., vol. 33, pp. 521-525, 2001.

[17] S.E. Derenzo, M.S. Boswell, E. Bourret-Courchesne, R. Boutchko, T. F. Budinger, A. Canning, S.M. Hanrahan, M. Janecek, Q Peng, Y. PorterChapman, J. Powell, C.A. Ramsey, S.E. Taylor, L.-W. Wang, M.J. Weber, and D.S. Wilson, "Design and Implementation of a Facility for Discovering New Scintillator Materials", IEEE Trans. Nucl. Sci., vol. 55, no. 3, pp. 1458-1463, Jun. 2008.

[18] M. Moszynski, M. Balcerzyk, W. Czarnacki, M. Kapusta, W. Klamra, P. Shotanus, A. Syntfeld, A. Szawlowski, "Study of pure NaI at room and liquid nitrogen", IEEE Trans. Nucl. Sci., vol. 50, no. 4, pp. 767-773, Aug. 2003.

[19] S.C. Blankespoor, S.E. Derenzo, W.W. Moses, and C.S. Rossington, "Characterization of a pulsed x-ray source for fluorescent lifetime measurements", IEEE Trans. Nucl. Sci., vol. NS-41, pp. 698-702, 2004.

[20] S. Kubota, F. Shiraishi and Y. Takami, "Decay curves of NaI(Tl) with different $\mathrm{Tl}^{+}$concentrations under excitation of electrons, alpha particles and fission fragments", J. Phys. Soc. Jpn., vol. 68, no. 1, pp. 291-297, Jan. 1998.. 